\title{
Resistência de clones de seringueira à infestação por ácaros
}

\author{
Marineide Rosa Vieira $\left({ }^{1 *}\right)$; Gustavo Luís Mamoré Martins $\left({ }^{2}\right)$; Erivaldo José Scaloppi Junior ( $\left.{ }^{3}\right)$ \\ (') Universidade Estadual Paulista (UNESP), Departamento de Fitossanidade, Engenharia Rural e Solos, 15385-000 Ilha Solteira (SP), \\ Brasil \\ (2) Universidade Estadual do Mato Grosso do Sul (UEMS), Unidade Universitária de Cassilândia, 79540-000 Cassilândia (MS), Brasil \\ (3) Agência Paulista de Tecnologia dos Agronegócios (APTA), Polo Regional do Noroeste Paulista, 15500-970 Votuporanga (SP), Brasil \\ (*) Autor correspondente: marineid@bio.feis.unesp.br
}

Recebido: 15/ago./2013; Aceito: 26/out./2013

\begin{abstract}
Resumo
Calacarus heveae Feres (Eriophyidae) e Tenuipalpus heveae Baker (Tenuipalpidae) podem causar intenso desfolhamento em plantas de seringueira. Na perspectiva de implantação de manejo integrado de pragas, o uso de clones resistentes pode vir a ser uma importante estratégia de controle. O objetivo deste trabalho foi avaliar a resistência de clones de seringueira ao ataque desses ácaros em experimento conduzido em Votuporanga, SP. O delineamento experimental foi o de blocos casualizados com 18 tratamentos e três repetições, sendo que os tratamentos adotados foram: clones PB 230, PB 243, PB 252, PB 294, PB 306, PB 311, PB 312, PB 314, PB 324, PB 346, PB 350, PB 355, IAC 56, IAC 302, IAC 328, IAC 334, Fx 3899 e RRIM 600. As avaliações populacionais de C. heveae e T. heveae foram realizadas pela contagem em laboratório dos ácaros e exúvias presentes em seis folíolos por parcela. Os níveis de desfolhamento foram avaliados com uma escala de notas de 0 a 4 , sendo 0 ausência de desfolhamento e 4 desfolhamento acima de 75\%. Com base nos resultados pode-se concluir que o clone Fx 3899 é resistente a C. heveae por não preferência e/ou antibiose. O clone IAC 56 é menos suscetível ao desfolhamento provocado pela infestação de C. heveae e T. heveae.
\end{abstract}

Palavras-chave: Hevea brasiliensis, Calacarus heveae, Tenuipalpus heveae, Acari.

\section{Resistance of rubber tree clones to mite infestation}

\section{Abstract}

Calacarus heveae Feres (Eriophyidae) and Tenuipalpus heveae Baker (Tenuipalpidae) can cause heavy defoliation of rubber tree plants. From the perspective of integrated pest management, resistant clones can be an important strategy. The objective of this work was to evaluate the resistance of rubber tree clones to the attack of mites, in an experiment carried out in Votuporanga, SP. The experimental design was a randomized blocks with eighteen treatments and three replications. The treatments were the clones PB 230, PB 243, PB 252, PB 294, PB 306, PB 311, PB 312, PB 314, PB 324, PB 346, PB 350, PB 355, IAC 56, IAC 302, IAC 328, IAC 334, Fx 3899 and RRIM 600. The evaluation of C. heveae and T. heveae populations was performed by counting mites and exuviae presence in six leaflets per plot. The defoliation levels were evaluated with a score scale, varying from 0 (no defoliation) to 4 (above 75\% of defoliation). Based on the results, Fx 3899 clone is resistant through non-preference and/or antibiosis to C. heveae. The IAC 56 clone is less susceptible to defoliation caused by C. heveae and T. heveae infestation.

Key words: Hevea brasiliensis, Calacarus heveae, Tenuipalpus heveae, Acari.

\section{INTRODUÇÃO}

A área cultivada com seringueira [Hevea brasiliensis (Willd. ex. Adr. de Juss.) Müell Arg] tem aumentado no estado de São Paulo, o maior produtor brasileiro de látex (GonçALVES, 2010). Essa expansão para novas áreas tem favorecido a infestação de ácaros-praga, como Calacarus heveae Feres (Eriophyidae) e Tenuipalpus heveae Baker (Tenuipalpidae) (Moraes e Flechtmann, 2008). Os ácaros infestam as folhas de seringueira causando amarelecimento, necroses e posterior desfolhamento, que pode atingir níveis acima de $75 \%$ em até dois meses antes do período de senescência normal (Gonçalves, 2010; Vieira e Gomes, 1999).

Dentro da perspectiva do manejo integrado de pragas, o uso de clones resistentes pode ser uma importante estratégia de controle. A resistência de clones às principais doenças tem sido contemplada em programas de melhoramento (GonÇAlves e Marques, 2008), mas são necessárias maiores informaçôes sobre a resistência às pragas. Três tipos de resistência de plantas a pragas podem ocorrer: não preferência, antibiose e tolerância. Uma variedade apresenta resistência do tipo não preferência quando é 
menos utilizada pela praga para alimentação, oviposição ou abrigo que outra em igualdade de condiçóes. Antibiose ocorre quando a variedade pode exercer um efeito adverso sobre a biologia da praga, enquanto tolerante é aquela que sofre poucos danos em relaçáo a outras sob um mesmo nível de infestação pela praga (Vendramim e Guzzo, 2009).

Clones resistentes aos ácaros que atacam a cultura da seringueira podem ser detectados pela avaliação do desenvolvimento populacional das espécies, que pode indicar a presença de fatores de resistência por não preferência e/ou por antibiose e pela avaliação do desfolhamento provocado, indicativo da resistência por tolerância (Silva et al., 2011). A presença de fatores de resistência a $C$. heveae e $T$. heveae em diferentes clones comerciais de seringueira tem sido avaliada por alguns autores (DAUd e FEREs, 2007; DAUd et al., 2012b; Feres et al., 2010; Ferla e Moraes, 2008; Silva et al., 2011). Resistência a esses ácaros já foi observada nos clones GT 1 e PB 217, para o estado de Mato Grosso (Daud e Feres, 2007), e nos clones IAC 15, IAC 40, IAC 300, IAN 3156, PB 28/59 e IRCA 111, para o estado de São Paulo (Silva et al., 2011). Em experimentos de laboratório, DAud et al. (2012b) comprovaram a maior resistência do clone GT 1 à infestação de $C$. heveae. O clone RRIM 600, de grande importância para a heveicultura, é suscetível à infestação das duas espécies (Daud e Feres, 2007; Daud et al., 2012a; Silva et al., 2011; Vieira e Gomes, 1999; Vieira et al., 2006).

$\mathrm{O}$ uso de clones resistentes pode reduzir os custos de produção com menor aplicação de acaricidas na heveicultura e diminuir as possibilidades de contaminação ambiental, preservando os agentes de controle biológico já identificados em seringueira (Hernandes e Feres, 2006b). Nesse contexto, o objetivo do trabalho foi avaliar a resistência de 18 clones de seringueira ao ataque de $C$. heveae e $T$. heveae no município de Votuporanga, SP.

\section{MATERIAL E MÉTODOS}

A pesquisa foi desenvolvida no Polo Regional do Noroeste Paulista, APTA, no município de Votuporanga, SP (latitude $20^{\circ} 20^{\prime} \mathrm{S}$, longitude $49^{\circ} 58^{\prime} \mathrm{W}$ e altitude de $510 \mathrm{~m}$ ), em 2007 , 2009 e 2010. A área experimental constituiu-se de plantas de 36 clones de seringueira, cultivadas em espaçamento $2,5 \times 8,0$ metros e com cinco anos de idade em 2007. Essas plantas estavam distribuídas segundo um delineamento experimental de blocos casualizados com três repetiçôes, sendo cada repetiçáo formada por uma linha de oito plantas. No primeiro ano foram avaliados os clones PB 230, PB 243, PB 252, PB 294, PB 306, PB 311, PB 312, PB 314, PB 324, PB 346, PB 350, PB 355, IAC 56, IAC 302, IAC 328, IAC 334, Fx 3899 e RRIM 600, totalizando 18 tratamentos. Com base nos resultados obtidos, foram selecionados oito clones para novas avaliaçôes, conduzidas em 2009 e 2010:
PB 294, PB 306, PB 312, PB 350, IAC 56, IAC 328, Fx 3899 e RRIM 600.

Duas amostragens foram realizadas nos dois primeiros anos, em 24/5/2007 e 22/6/2007 e em 24/4/2009 e 4/6/2009. Em 2010, as avaliaçóes foram realizadas em 18/2, 31/3 e $18 / 5$, além de uma avaliaçáo final de desfolhamento em 29/6. Em cada data, de cada parcela foram coletadas, de pontos diferentes, com auxílio de uma tesoura de poda alta, duas extremidades de ramos com aproximadamente $30 \mathrm{~cm}$ de comprimento. Os ramos foram acondicionados em sacos plásticos e encaminhados ao laboratório para identificação e contagem de ácaros e exúvias.

Em laboratório, cada espécie de ácaro foi avaliada em seis folíolos (três de cada extremidade de ramo) de cada parcela. A avaliação de $C$. heveae foi realizada em duas áreas de $1 \mathrm{~cm}^{2}$, na página superior de cada folíolo, uma de cada lado da nervura principal. Para T. heveae foram examinadas duas áreas de $1 \mathrm{~cm}^{2}$ por folíolo, na página inferior, uma sobre a nervura principal e uma sobre uma nervura lateral. Em cada área foi realizada a contagem de ácaros e exúvias. Devido à grande quantidade de exúvias presentes nos folíolos e considerando-se que o objetivo era identificar clones com baixo nível populacional dos ácaros, elas foram contadas até o valor máximo de 400 por $\mathrm{cm}^{2}$, sendo esse valor atribuído a todas as áreas com ocorrência superior de exúvias.

O nível de desfolhamento observado nas plantas foi avaliado com o uso de uma escala de notas de 0 a 4 , sugerida por Vieira e Gomes (1999), sendo 0 ausência de desfolhamento, 1 para $0 \%$ a $25 \%$ de desfolhamento, 2 para $25 \%$ a $50 \%, 3$ para $50 \%$ a $75 \%$ e 4 para desfolhamento acima de $75 \%$.

Os dados de número médio de ácaros e exúvias por $\mathrm{cm}^{2}$ e notas de desfolhamento foram submetidos à análise de variância e as médias foram agrupadas pelo teste de Scott-Knott a 5\% de significância. No caso de ácaros e exúvias, os dados foram transformados em $\log (x+2)$, por não apresentarem aderência à normalidade (teste de Lilliefors), considerando-se, para as notas de desfolhamento, os dados originais. Os números de exúvias por $\mathrm{cm}^{2}$ registrados em cada ano, para cada espécie, foram comparados pelo teste $t$ de Student.

Os dados climáticos de temperatura e precipitação pluviométrica foram coletados no posto meteorológico do Polo Regional do Noroeste Paulista, APTA, Votuporanga, SP.

Para a seleçáo dos clones a serem avaliados em 2009 e 2010, o clone RRIM 600, o mais plantado no estado de São Paulo, foi considerado o padrão de comparação. Assim, foram considerados suscetíveis os clones que apresentaram nota média de desfolhamento maior que a registrada em RRIM 600. Também foi considerado suscetível o clone em que o número médio de ácaros e/ou exúvias de pelo menos uma das espécies foi maior que o observado no clone padrão. Clones suscetíveis não foram avaliados novamente. 
Para a classificaçáo dos clones foi considerado como indicativo da presença de resistência por não preferência e/ou antibiose o registro de número médio de ácaros e/ou exúvias inferior ao registrado em RRIM 600, nos três períodos avaliados.

\section{RESULTADOS E DISCUSSÃO}

Houve diferença significativa entre os clones quanto ao desenvolvimento populacional das duas espécies consideradas, expresso pelo número médio de ácaros e de exúvias (Tabelas 1 e 2). No ano de 2007 (Tabela 1), nos clones PB 230, PB 252, PB 355 e IAC 302, o número médio de C. heveae foi superior ao observado em RRIM 600, enquanto nos outros clones esse número não diferiu desse padrão. Os clones náo diferiram em relação à densidade de exúvias presentes nos folíolos, com exceção do clone Fx 3899, que apresentou a menor média. Para T. heveae, o número médio de ácaros foi semelhante em todos os clones, mas a quantidade de exúvias foi menor nos clones PB 230, PB 243, PB 252, PB 294, PB 355, Fx 3899, IAC 56 e IAC 328. No clone RRIM 600, a nota média de desfolhamento foi 1,7 , não diferindo das registradas nos clones PB 294, PB 306, PB 312, PB 350, PB 355 e IAC 302. As menores notas para queda de folhas foram observadas nos clones Fx 3899, IAC 56 e IAC 328. Nos demais clones, a nota de desfolhamento foi maior do que em RRIM 600.

Com base nesses resultados foi realizada a seleçáo dos clones para serem avaliados em 2009 e 2010. Foram selecionados para uma nova avaliação: clone Fx 3899, devido ao menor número de exúvias das duas espécies e ao menor desfolhamento; clones IAC 56 e IAC 328, que apresentaram menor quantidade de exúvias de $T$. heveae e menor nível de desfolhamento; PB 294, com menor nível de exúvias de T. heveae. Além deles, também foram incluídos os clones PB 306, PB 312 e PB 350, que apresentaram comportamento semelhante ao do RRIM 600.

Em 2009, os clones não diferiram quanto ao número médio de ácaros das duas espécies (Tabela 2). Entretanto, quanto às exúvias, no clone PB 350 foi registrado menor número das de $C$. heveae e, em Fx 3899, menor quantidade das dos dois ácaros. O menor desfolhamento foi observado nos clones Fx 3899, IAC 56 e IAC 328, com nota 1,0. Nos clones PB 306 e PB 350, a queda de folhas não diferiu da ocorrida no RRIM 600.

No terceiro ano de avaliação, os clones PB 294, PB 306, PB 312, PB 350 e IAC 328 não diferiram do clone padrão quanto à densidade de ácaros e exúvias e ao desfolhamento das plantas (Tabela 2). Em 2010, no clone Fx 3899, o número médio de exúvias de $C$. heveae e a nota média de desfolhamento das plantas foram inferiores aos registrados em RRIM 600, enquanto o número de exúvias de $T$. heveae não diferiu desse controle.
Tabela 1. Número médio ${ }^{(1)}$ de ácaros e exúvias, por $\mathrm{cm}^{2}$ de folíolo, de Calacarus heveae e Tenuipalpus heveae e nota média ${ }^{(2),(3)}$ de desfolhamento em clones de seringueira, em Votuporanga, SP, 2007

\begin{tabular}{|c|c|c|c|c|c|}
\hline \multirow[t]{2}{*}{ Clones } & \multicolumn{2}{|c|}{ C. heveae } & \multicolumn{2}{|c|}{ T. heveae } & \multirow{2}{*}{$\begin{array}{c}\text { Notas de } \\
\text { desfolhamento }\end{array}$} \\
\hline & Ácaros & Exúvias & Ácaros & Exúvias & \\
\hline PB 230 & $8,9 a$ & 343,8 a & $0,7 \mathrm{a}$ & $26,1 \mathrm{~b}$ & $3,4 a$ \\
\hline PB 243 & $3,6 \mathrm{~b}$ & 308,0 a & $0,2 \mathrm{a}$ & $16,9 \mathrm{~b}$ & $2,8 \mathrm{a}$ \\
\hline PB 252 & $4,9 a$ & 307,6 a & $0,6 \mathrm{a}$ & $35,3 \mathrm{~b}$ & $2,4 \mathrm{~b}$ \\
\hline PB 294 & $1,1 \mathrm{~b}$ & 331,6 a & $0,4 \mathrm{a}$ & $16,4 \mathrm{~b}$ & $1,7 \mathrm{c}$ \\
\hline PB 306 & $0,3 \mathrm{~b}$ & 200,6 a & $0,1 \mathrm{a}$ & $43,7 \mathrm{a}$ & $1,7 \mathrm{c}$ \\
\hline PB 311 & $0,2 \mathrm{~b}$ & 368,9 a & $0,7 \mathrm{a}$ & $36,6 \mathrm{a}$ & $2,2 \mathrm{~b}$ \\
\hline PB 312 & $1,5 \mathrm{~b}$ & $383,2 \mathrm{a}$ & $0,4 \mathrm{a}$ & $35,4 \mathrm{a}$ & $1,5 \mathrm{c}$ \\
\hline PB 314 & $0,1 \mathrm{~b}$ & 287,9 a & $0,4 \mathrm{a}$ & $55,6 \mathrm{a}$ & $2,6 \mathrm{~b}$ \\
\hline PB 324 & $1,4 \mathrm{~b}$ & $307,2 \mathrm{a}$ & $0,5 \mathrm{a}$ & $46,4 \mathrm{a}$ & $2,2 \mathrm{~b}$ \\
\hline PB 346 & $1,1 \mathrm{~b}$ & 369,4 a & $0,2 \mathrm{a}$ & $62,1 \mathrm{a}$ & $2,2 \mathrm{~b}$ \\
\hline PB 350 & $0,2 \mathrm{~b}$ & $321,9 \mathrm{a}$ & $0,4 \mathrm{a}$ & $37,3 \mathrm{a}$ & $2,0 \mathrm{c}$ \\
\hline PB 355 & $21,6 \mathrm{a}$ & 381,6 a & $3,8 \mathrm{a}$ & $14,9 \mathrm{~b}$ & $2,0 \mathrm{c}$ \\
\hline Fx 3899 & $0,1 \mathrm{~b}$ & $142,6 \mathrm{~b}$ & $1,5 \mathrm{a}$ & $18,2 b$ & $1,0 \mathrm{~d}$ \\
\hline IAC 56 & $0,3 \mathrm{~b}$ & $251,6 \mathrm{a}$ & $0,2 \mathrm{a}$ & $26,0 \mathrm{~b}$ & $1,1 \mathrm{~d}$ \\
\hline IAC 302 & $16,5 \mathrm{a}$ & 279,4 a & $0,6 \mathrm{a}$ & $87,2 \mathrm{a}$ & $1,8 \mathrm{c}$ \\
\hline IAC 328 & $0,7 \mathrm{~b}$ & 364,1 a & $0,1 \mathrm{a}$ & $15,5 \mathrm{~b}$ & $1,2 \mathrm{~d}$ \\
\hline IAC 334 & $3,2 \mathrm{~b}$ & $342,8 \mathrm{a}$ & $2,0 \mathrm{a}$ & $105,7 \mathrm{a}$ & $2,9 \mathrm{a}$ \\
\hline RRIM 600 & $0,6 \mathrm{~b}$ & $314,2 \mathrm{a}$ & $0,4 \mathrm{a}$ & $45,2 \mathrm{a}$ & $1,7 \mathrm{c}$ \\
\hline CV (\%) & 57,9 & 9,1 & 39,9 & 23,9 & 27,7 \\
\hline
\end{tabular}

Médias seguidas pela mesma letra na coluna pertencem ao mesmo agrupamento, pelo teste de Scott-Knott a 5\% de significância; ${ }^{(1)}$ Médias originais; para análise estatística, os dados foram transformados em $\log (\mathrm{x}+2)$; ${ }^{(2)}$ Médias originais; análise dos dados sem transformação; ${ }^{(3)}$ Escala de notas: $0=$ ausência de desfolhamento; $1=0 \%$ a $25 \%$ de desfolhamento; $2=25 \%$ a $50 \%$ de desfolhamento; $3=50 \%$ a $75 \%$ de desfolhamento; e 4 = acima de $75 \%$ de desfolhamento

Parte do desfolhamento observado na área pode ter ocorrido em função de um processo natural de senescência. Entretanto, nos três anos de estudo foi observada grande quantidade de folíolos com sintomas de ataque das duas espécies nas plantas e no chão da área, indicando ser essa a principal causa do desfolhamento.

A avaliação de genótipos quanto à infestação de $C$. heveae e T. heveae tem sido feita com base no número médio de ácaros registrado em todo o período de produção da cultura, do enfolhamento à senescência (SiLVA et al., 2011; VieIRA et al. 2009). Uma vez que o período de maior ocorrência das espécies pode ir de fevereiro a junho (Hernandes e Feres, 2006a; Vieira e Gomes, 1999; Vieira et al., 2006; Vis et al., 2006), o número de ácaros poderia não refletir corretamente o nível de infestação, caso as maiores populaçôes tivessem ocorrido em período anterior ao avaliado (no presente trabalho, duas avaliaçóes no período de abril a junho). Como as exúvias são depositadas na superfície foliar e aí permanecem (Feres, 1992), o número de exúvias foi considerado, buscando refletir o nível real de infestação ocorrido em cada clone.

Vieira e Gomes (1999), com base no clone RRIM 600, definiram o nível de 0,94 ácaros por $\mathrm{cm}^{2}$ como o limite acima do qual $C$. heveae pode provocar desfolhamento das plantas. No presente trabalho, esse nível só não foi superado 


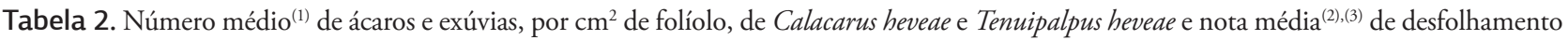
em clones de seringueira, em Votuporanga, SP, 2009 e 2010

\begin{tabular}{|c|c|c|c|c|c|c|c|c|c|c|c|c|}
\hline \multirow[t]{3}{*}{ Clones } & \multicolumn{5}{|c|}{2009} & \multicolumn{5}{|c|}{2010} & \multirow{2}{*}{\multicolumn{2}{|c|}{ Resistência ${ }^{(4)}$}} \\
\hline & \multicolumn{2}{|c|}{ C. heveae } & \multicolumn{2}{|c|}{ T. heveae } & \multirow[t]{2}{*}{ Nota } & \multicolumn{2}{|c|}{ C. heveae } & \multicolumn{2}{|c|}{ T. heveae } & \multirow[t]{2}{*}{ Nota } & & \\
\hline & Ác. & Ex. & Ác. & Ex. & & Ác. & Ex. & Ác. & Ex. & & Ch & Th \\
\hline PB 294 & $1,8 \mathrm{a}$ & $189,8 \mathrm{a}$ & $0,2 \mathrm{a}$ & $8,4 \mathrm{a}$ & $2,2 \mathrm{a}$ & $0,7 \mathrm{a}$ & $133,2 \mathrm{a}$ & $0,1 \mathrm{a}$ & $2,7 \mathrm{a}$ & $3,8 \mathrm{a}$ & $\mathrm{S}$ & $S$ \\
\hline PB 306 & $0,5 \mathrm{a}$ & $162,7 \mathrm{a}$ & $0,1 \mathrm{a}$ & $7,0 \mathrm{a}$ & $1,7 \mathrm{~b}$ & $0,4 \mathrm{a}$ & $126,9 a$ & $0,3 \mathrm{a}$ & $1,9 a$ & $4,0 \mathrm{a}$ & $\mathrm{S}$ & $\mathrm{S}$ \\
\hline PB 312 & $1,4 \mathrm{a}$ & $177,0 \mathrm{a}$ & $0,1 \mathrm{a}$ & $7,3 \mathrm{a}$ & $2,5 \mathrm{a}$ & $1,3 \mathrm{a}$ & $141,8 \mathrm{a}$ & $0,2 \mathrm{a}$ & $2,0 \mathrm{a}$ & $4,3 \mathrm{a}$ & $\mathrm{S}$ & $\mathrm{S}$ \\
\hline PB 350 & $2,1 \mathrm{a}$ & $131,4 \mathrm{~b}$ & $0,2 \mathrm{a}$ & $5,3 \mathrm{a}$ & $1,7 \mathrm{~b}$ & $0,1 \mathrm{a}$ & $107,1 \mathrm{a}$ & $0,2 \mathrm{a}$ & $1,6 \mathrm{a}$ & $3,7 \mathrm{a}$ & $\mathrm{S}$ & $\mathrm{S}$ \\
\hline Fx 3899 & $0,9 \mathrm{a}$ & $27,5 \mathrm{c}$ & $0,1 \mathrm{a}$ & $1,1 \mathrm{~b}$ & $1,0 \mathrm{c}$ & $0,3 \mathrm{a}$ & $19,4 \mathrm{~b}$ & $0,2 \mathrm{a}$ & $1,6 \mathrm{a}$ & $2,0 \mathrm{c}$ & $\mathrm{R}_{\mathrm{NP} / \mathrm{A}}$ & $\mathrm{R}_{\mathrm{NP} / \mathrm{A}}$ \\
\hline IAC 56 & $1,7 \mathrm{a}$ & $180,3 \mathrm{a}$ & $0,1 \mathrm{a}$ & $8,0 \mathrm{a}$ & $1,0 \mathrm{c}$ & $1,3 \mathrm{a}$ & $132,8 \mathrm{a}$ & $0,1 \mathrm{a}$ & $0,5 \mathrm{a}$ & $2,9 \mathrm{~b}$ & MSd & MSd \\
\hline IAC 328 & $1,4 \mathrm{a}$ & $173,2 \mathrm{a}$ & $0,2 \mathrm{a}$ & $8,2 \mathrm{a}$ & $1,0 \mathrm{c}$ & $0,3 \mathrm{a}$ & $112,4 a$ & $0,1 \mathrm{a}$ & $0,4 \mathrm{a}$ & $3,7 \mathrm{a}$ & $\mathrm{S}$ & $\mathrm{S}$ \\
\hline RRIM 600 & 3,9 a & $173,1 \mathrm{a}$ & $0,3 \mathrm{a}$ & $8,4 \mathrm{a}$ & $1,5 \mathrm{~b}$ & $0,6 \mathrm{a}$ & $136,5 \mathrm{a}$ & $0,1 \mathrm{a}$ & $1,1 \mathrm{a}$ & $3,5 \mathrm{a}$ & $S$ & $S$ \\
\hline CV (\%) & 26,1 & 5,4 & 12,1 & 18,7 & 28,5 & 34,8 & 23,6 & 16,0 & 39,3 & 12,0 & & \\
\hline
\end{tabular}

Médias seguidas pela mesma letra na coluna pertencem ao mesmo agrupamento, pelo teste de Scott-Knott a 5\% de significância; ${ }^{(1)}$ Médias originais; para análise estatística, os dados foram transformados em $\log (\mathrm{x}+2) ;{ }^{(2)}$ Médias originais; análise dos dados sem transformaçâo; ${ }^{(3)}$ Escala de notas: 0 = ausência de desfolhamento; 1 = $0 \%$ a $25 \%$ de desfolhamento; $2=25 \%$ a $50 \%$ de desfolhamento; $3=50 \%$ a $75 \%$ de desfolhamento; e 4 = acima de $75 \%$ de desfolhamento; ${ }^{(4)}$ S: suscetível; $\mathrm{R}_{\mathrm{NP} / \mathrm{A}}$ : resistência por năo preferência e/ou antibiose; MSd: menor suscetibilidade ao desfolhamento

nos clones PB 311 e PB 314. Nos demais clones, o nível populacional de $C$. heveae ultrapassou esse valor em pelo menos uma das amostragens. No clone RRIM 600, em 24/4/2009, a população de $C$. heveae atingiu o nível de 4,2 ácaros $\mathrm{cm}^{-2}$.

Pela contagem do número de exúvias foi possível verificar que a infestaçáo de $C$. heveae foi maior em 2007 do que em $2009(\mathrm{t}=3,9134, \mathrm{p}=0,0016)$ e $2010(\mathrm{t}=4,4445, \mathrm{p}=0,0001)$, o mesmo ocorrendo para $T$. heveae em relação ao segundo $(\mathrm{t}=5,2026, \mathrm{p}=0,0012)$ e terceiro $(\mathrm{t}=6,3595, \mathrm{p}=0,0001)$ anos de avaliaçáo. Entre os fatores que podem ter contribuído para essa diferença está a ocorrência de precipitaçōes. De agosto de 2006 a junho de 2007 foi registrada precipitação total de $1.578,8 \mathrm{~mm}$, com pico de $618,6 \mathrm{~mm}$ no mês de janeiro (Figura 1). Por outro lado, de agosto de 2008 a junho de 2009 , a precipitaçáo foi de apenas $990,8 \mathrm{~mm}$. Maiores infestaçôes das duas espécies em ano mais chuvoso também foram registradas em Selvíria, MS (Vieira et al., 2009). Para C. heveae, a maior população registrada em 2007 era esperada, uma vez que esse ácaro é favorecido por ambiente úmido, conforme os dados de Ferla e Moraes (2003), os quais verificaram, em estudos de laboratório, a viabilidade da fase de ovo a adulto acima de $90 \%$, com umidade relativa do ar de $90 \pm 5 \%$. Por outro lado, a ocorrência de $T$. heveae, no estado de São Paulo, tem sido associada ao fim do período chuvoso, em Olímpia (Bellini et al., 2005) e Piracicaba (Vis et al., 2006). Também em Cedral, SP, Hernandes e Feres (2006a), em três anos de levantamento populacional, registraram maior população de $T$. heveae no ano menos chuvoso. Dessa forma, é possível que outros fatores tenham contribuído para a menor infestação dessa espécie em 2009. DAuD et al. (2012b) observaram que folhas de seringueira podem variar quanto à sua adequação como hospedeiras de ácaros de acordo com a época do ano. Segundo esses autores, em experimentos de laboratório, o melhor desenvolvimento de $C$. heveae ocorreu quando o estudo foi conduzido em folhas

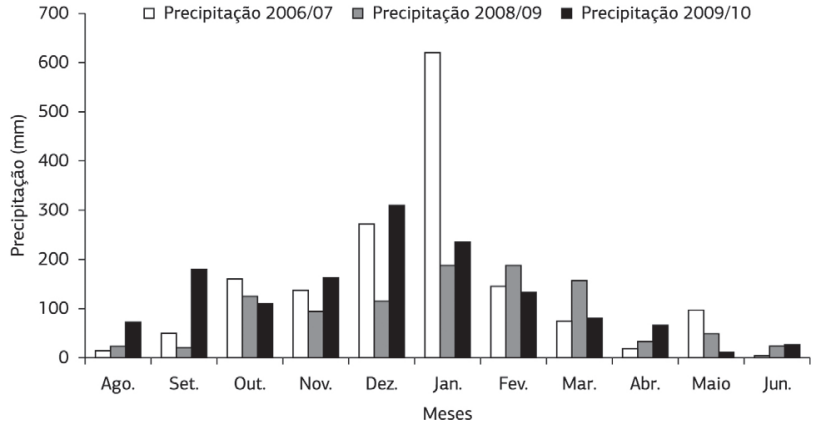

Figura 1. Precipitaçáo total registrada nos meses de agosto a junho nos períodos 2006-2007, 2008-2009 e 2009-2010 no posto meteorológico do Polo Regional do Noroeste Paulista, APTA, Votuporanga, SP.

coletadas no período de janeiro a abril, em comparaçáo com folhas trazidas do campo nos períodos de novembro/dezembro e maio/junho, demonstrando a existência de diferenças na condição fisiológica das plantas que podem afetar a acarofauna. No terceiro período, de agosto de 2009 a junho de 2010, as chuvas totalizaram $1.392,9 \mathrm{~mm}$. Embora esse valor seja próximo do registrado em 2006/2007, a distribuição foi diferente (Figura 1), o que pode ter afetado as populaçóes de ácaros. O efeito da fisiologia das plantas, das condiçóes climáticas e de outros fatores ambientais permanece como uma importante linha de pesquisa para o futuro.

Para que um clone possa ser considerado resistente, é necessário que haja repetibilidade do comportamento observado, sempre que mantidas as mesmas condiçóes em que ele foi registrado (Vendramim e Guzzo, 2009). Neste trabalho, um clone foi considerado resistente quando o comportamento de resistência foi observado nos três períodos estudados.

Dessa forma, os números de exúvias registrados em 2007, 2009 e 2010 para o clone Fx 3899 são indicativos da presença de resistência a $C$. heveae. Esses resultados 
foram significativamente diferentes dos registrados no clone RRIM 600 nos três períodos de avaliação. Por outro lado, para T. heveae, a densidade de ácaros e exúvias em 2010 não diferiu da registrada no clone padrão. Entretanto, no terceiro ano existe a possibilidade de que o nível populacional da espécie, pequeno em relação aos outros anos, não tenha sido suficiente para permitir a discriminaçáo dos clones e, portanto, a suscetibilidade a $T$. heveae necessita de nova avaliação. A resistência de uma planta ao desenvolvimento populacional de uma praga pode ser atribuída a um comportamento de não preferência para alimentação, oviposição ou abrigo ou a um efeito de antibiose, quando a planta afeta a biologia da praga (Vendramim e Guzzo, 2009). Não é possível determinar qual dos dois tipos de resistência está envolvido no desempenho observado em relação à infestação de $C$. heveae, ou mesmo se os dois tipos participam do processo. A não aceitação desse clone como alimento ou abrigo pode ter levado os ácaros a um comportamento de fuga, mas o efeito de antibiose também é possível, uma vez que a presença de substâncias deletérias no substrato alimentar pode afetar o potencial reprodutivo dos ácaros (TADMOR et al., 1999; VIEIRA et al., 2002). Assim, o menor desfolhamento poderia ser atribuído ao menor desenvolvimento dos ácaros.

No clone IAC 56, o número de ácaros e de exúvias de $C$. heveae não diferiu dos registrados em RRIM 600. Entretanto, nos três anos, o desfolhamento em IAC 56 foi inferior ao desse padrão, indicando menor suscetibilidade à infestação de $C$. heveae. Em IAC 56, o número de exúvias de T. heveae foi menor em 2007, mas o mesmo não foi observado nos anos seguintes e, portanto, foi considerado semelhante ao clone RRIM 600 quanto ao desenvolvimento da espécie e, devido ao menor desfolhamento, menos suscetível aos danos da infestação.

Resistência aos ácaros da cultura foi registrada por Silva et al. (2011) na mesma unidade de pesquisa da APTA em Votuporanga. Os autores concluíram que os clones IAC 15, IAC 40, IAC 300, IAN 3156 e PB 28/59 apresentam resistência por não preferência e/ou antibiose a $C$. heveae e $T$. heveae e consideraram o clone IRCA 111 como tolerante ao ataque de $C$. heveae.

Dos clones avaliados no presente trabalho, apenas RRIM 600 está incluído na classe I de recomendação de plantio para o estado de São Paulo para utilizaçâo em grande escala. Por outro lado, os clones IAC 302, PB 252 e PB 350 estão na classe II, para plantio em escala moderada, enquanto os clones PB 311, PB 312, PB 314 e PB 355 são recomendados para plantio em escala experimental (IAC, 2013).

Neste trabalho, o clone RRIM 600 foi considerado o padrão de comparação devido à sua grande importância para a heveicultura paulista. Uma vez que esse clone apresenta suscetibilidade a ácaros, os quais podem provocar acentuado desfolhamento precoce (DAUd e FerEs, 2007; Silva et al., 2011; Vieira e Gomes, 1999; Vieira et al., 2006), as pesquisas devem ter por objetivo identificar clones com nível de resistência maior do que o observado em RRIM 600 e com, no mínimo, igual produtividade.

Dessa forma, pela redução de custos econômicos e ambientais em que o plantio de um clone mais resistente pode resultar, em função da redução da necessidade de pulverizaçóes, é oportuna a melhor avaliação da produtividade dos clones IAC 56 e Fx 3899 nas diversas condiçóes climáticas e de solo existentes nas áreas de plantio do estado.

\section{CONCLUSÃO}

O clone Fx 3899 é resistente a $C$. heveae por não preferência e/ou antibiose. O clone IAC 56 é menos suscetível ao desfolhamento provocado pela infestaçáo de $C$. heveae e T. heveae.

\section{REFERÊNCIAS}

BELLINI, M.R.; MORAES, G.J.; FERES, R.J.F. Ácaros (Acari) de dois sistemas de cultivo da seringueira no noroeste do estado de São Paulo. Neotropical Entomology, v.34, p.475-484, 2005. http://dx.doi. org/10.1590/S1519-566X2005000300017

DAUD, R.D.; FERES, R.J.F. Dinâmica populacional de ácaros fitófagos (Acari, Eriophyidae, Tenuipalpidae) em seis clones de seringueira no sul do Estado de Mato Grosso. Revista Brasileira de Entomologia, v.51, p.377-381, 2007. http://dx.doi.org/10.1590/ S0085-56262007000300016

DAUD, R.D.; CONFORTO, E.C.; FERES, R.J.F. Changes in leaf physiology caused by Calacarus heveae (Acari, Eriophyidae) on rubber tree. Experimental and Applied Acarology, v.57, p.127-137, 2012a. PMid:22527832. http://dx.doi.org/10.1007/s10493-012-9552-y

DAUD, R.D.; FERES, R.J.F.; HERNANDES, F.A. Seasonal suitability of three rubber tree clones to Calacarus heveae (Acari, Eriophyidae). Experimental and Applied Acarology, v.56, p.57-68, $2012 \mathrm{~b}$. PMid:21972083. http://dx.doi.org/10.1007/s10493-011-9494-9

FERES, J.F.F. A new species of Calacarus Keifer (Acari, Eriophyidae, Phyllocoptinae) from Hevea brasiliensis Muell. Arg. (Euphorbiaceae) from Brazil. International Journal of Acarology, v.18, p.61-65, 1992. http://dx.doi.org/10.1080/01647959208683930

FERES, R.J.F.; DEL'ARCO, M.; DAUD, R.D. Biological cycle of Tenuipalpus heveae Baker (Acari, Tenuipalpidae) on leaflets of three rubber tree clones. Revista Brasileira de Entomologia, v.54, p.298303, 2010. http://dx.doi.org/10.1590/S0085-56262010000200013

FERLA, N.J.; MORAES, G.J. Ciclo biológico de Calacarus heveae Feres, 1992. Revista Brasileira de Entomologia, v.47, p.399-402, 2003. http://dx.doi.org/10.1590/S0085-56262003000300006

FERLA, N.J.; MORAES, G.J. Flutuação populacional e sintomas de dano por ácaros (Acari) em seringueira no Estado do Mato Grosso, Brasil. Revista Árvore, v.32, p.365-376, 2008. http://dx.doi. org/10.1590/S0100-67622008000200019 
GONÇALVES, E.C.P. (Coord.). A cultura da seringueira para o Estado de São Paulo. 2. ed. Campinas: CATI, 2010. p.83-101. (Manual Técnico, n.72).

GONÇALVES, P.S.; MARQUES, J.R.B. Melhoramento genético da seringueira: passado, presente e futuro. In: ALVARENGA, A.P.; CARMO, C.A.F.S. (Coord.). Seringueira. Viçosa: EPAMIG, 2008. p.179-247.

HERNANDES, F.A.; FERES, R.J.F. Diversidade e sazonalidade de ácaros (Acari) em seringal (Hevea brasiliensis, Muell. Arg.) na regiáo noroeste do estado de São Paulo, Brasil. Neotropical Entomology, v.35, p.523-535, 2006a. PMid:17061803. http://dx.doi.org/10.1590/ S1519-566X2006000400016

HERNANDES, F.A.; FERES, R.J.F. Review about mites (Acari) of rubber trees (Hevea spp., Euphorbiaceae) in Brazil. Biota Neotropica, v.6, n.1, 2006b. Disponível em: <http://www.biotaneotropica.org.br/ v6n1/pt/item?article>. Acesso em: 14 ago. 2013.

INSTITUTO AGRONÔMICO - IAC. Programa seringueira: clones. 2013. Disponível em: <http://www.iac.sp.gov.br/areasdepesquisa/ seringueira/clones.php>. Acesso em: 31 out. 2013.

MORAES, G.J.; FLECHTMANN, C.H.W. Manual de Acarologia: acarologia básica e ácaros de plantas cultivadas no Brasil. Ribeirão Preto: Holos, 2008. 288p.

SILVA, H.A.S.; VIEIRA, M.R.; VALÉRIO FILHO, W.V.; CARDOSO, M.S.M.; FIGUEIRA, J.C. Clones de seringueira com resistência a ácaros. Bragantia, v.70, p.383-388, 2011. http://dx.doi.org/10.1590/ S0006-87052011000200019

TADMOR, Y.; LEWINSOHN, E.; ABO-MOCH, F.; BAR-ZUR, A.; MANSOUR, E. Antibiosis of maize inbred lines to the carmine spider mite, Tetranychus cinnabarinus. Phytoparasitica, v.27, p.35-41, 1999. http://dx.doi.org/10.1007/BF02980725

VENDRAMIM, J.D.; GUZZO, E.C. Resistência de plantas e a bioecologia e nutrição dos insetos. In: PANIZZI, A.R.; PARRA, J.R. (Ed.). Bioecologia e nutrição de insetos: base para o manejo integrado de pragas. Brasília: Embrapa Informação Tecnológica, 2009. p.1055-1105.

VIEIRA, M.R.; CAMPOS, A.R.; CASTRO, T.M.M.G.; SILVA, H.A.S.; FIGUEIRA, J.C.; MONTEVERDE, M.S. Resistência de cultivares de algodoeiro ao ácaro branco Polyphagotarsonemus latus (Banks) (Acari: Tarsonemidae). 1. Experimentos em laboratório. Revista Brasileira de Oleaginosas e Fibrosas, v.6, p.545-555, 2002.

VIEIRA, M.R.; GOMES, E.C. Sintomas, desfolhamento e controle de Calacarus heveae Feres, 1992 (Acari: Eriophyidae) em seringueira. Cultura Agronômica, v.8, p.53-71, 1999.

VIEIRA, M.R.; GOMES, E.C.; FIGUEIRA, J.C. Controle químico de Calacarus heveae Feres (Acari: Eriophyidae) em seringueira. Bioassay, v.1, p.1-7, 2006. Disponível em: <http://www.bioassay.org.br/bioassay/ article/view/37>. Acesso em: 14 ago. 2013.

VIEIRA, M.R.; SILVA, H.A.S.; CARDOSO, M.M.; FIGUEIRA, J.C. Progênies de seringueira com potencial para conferir resistência a ácaros (Calacarus heveae Feres e Tenuipalpus heveae Baker). Ciência Rural, v.39, p.1953-1959, 2009. http://dx.doi.org/10.1590/S010384782009005000164

VIS, R.M.J.; MORAES, G.J.; BELLINI, M.R. Mites (Acari) of rubber trees (Hevea brasiliensis Muell. Arg., Euphorbiaceae) in Piracicaba, State of São Paulo, Brazil. Neotropical Entomology, v.35, p.112120, 2006. PMid:17352076. http://dx.doi.org/10.1590/S1519566X2006000100015 